\title{
Repetitive Transcranial Magnetic Stimulation: A Call for Better Data
}

\author{
Marcus T. Wilson * and Lynley St George \\ School of Engineering, University of Waikato, Hamilton, New Zealand
}

Keywords: repetitive transcranial magnetic stimulation (rTMS), principal components analysis (PCA), motor evoked potential (MEP), synaptic plasticity (LTP/LTD), correlation analysis

\section{INTRODUCTION}

Repetitive Transcranial Magnetic Stimulation (rTMS) is increasingly used to treat stroke, Parkinson's disease and depression (Fregni et al., 2005; Loo and Mitchell, 2005; Hallet, 2007; O'Reardon et al., 2007; Ridding and Rothwell, 2007). rTMS uses bursts of magnetic pulses to change the excitability and connection strengths of cortical neurons. However, the evidence to inform clinical application is highly inconsistent (Thut and Pascual-Leone, 2010; Hamada et al., 2013) and substantially based on trial and error. Systematic theory is lacking. Typically, in rTMS research, measurements of motor-evoked potential (MEP) are made, often in terms of the strength of the MEP and the length of the cortical silent period that follows. However, the MEP is probably a poor and certainly an indirect measure of changes in the brain (Nicolo et al., 2015), clouding our understanding of rTMS mechanisms. In practice, therefore, particular amplitudes and timing of pulses in an rTMS sequence are selected because they show promise in small subsets of people. However, even basics such as the sign of any change in the outcome measure (e.g., does the MEP increase or decrease?) is debated. Many results show a wide spread in responses. It has become common to talk about "responders" and "non-responders" although evidence for a binary

OPEN ACCESS

Edited by: Jennifer Rodger University of Western Australia, Australia

Reviewed by:

Simon C. Gandevia, Neuroscience Research Australia, Australia

Charles P. Lewis,

Mayo Clinic, USA

${ }^{*}$ Correspondence:

Marcus T. Wilson mtwilson@waikato.ac.nz

Received: 13 May 2016

Accepted: 20 July 2016 Published: 03 August 2016

Citation:

Wilson MT and St George L (2016) Repetitive Transcranial Magnetic Stimulation: A Call for Better Data. Front. Neural Circuits 10:57. doi: 10.3389/fncir.2016.00057 distinction in these two groups is lacking-in reality there is usually a continuum of response often including potentiation in some and depression in others (Nettekoven et al., 2015). Moreover, Héroux et al. (2015) provide evidence that the irreproducibility of results may be due to small sample sizes, unscientific screening of subjects and data, and selective reporting of results.

In rTMS a regular train of pulses is applied. There is considerable variation in possible stimulation sequences, leading to many parameters that could potentially affect the results. Pulses are applied at a particular amplitude (normally recorded as percent motor threshold, (\%RMT), or sometimes percent machine output, themselves imprecise measures), at a given rate (pulses per second, or hertz, Hz), until a particular number of pulses have been applied. There are three numerical parameters here. Additionally, one can consider variation in coil shape, orientation and place of application. Different waveforms for the magnetic pulse are also available. Thus, for an ostensibly straightforward pulse sequence such as non-bursting rTMS, the parameter space is considerable. Fitzgerald et al. (2006) carried out a comprehensive review of rTMS effects. They concluded that low frequency $(0.9-5 \mathrm{~Hz})$ stimulation generally results in a decreased MEP, while high frequency stimulation $(5-20 \mathrm{~Hz})$ results in an increased MEP. Very low frequency stimulation gave no effect. The " $<5 \mathrm{~Hz}$, depression; $>5 \mathrm{~Hz}$, potentiation" mantra is now well established in rTMS literature.

More recently, bursting protocols have become popular. The quadripulse protocol (Tsutsumi et al., 2014) is one example-four pulses are applied in quick succession, with this pattern repeated at regular time intervals. Theta-burst stimulation (TBS) is another; bursts of pulses are applied at theta-band $(4-8 \mathrm{~Hz})$ frequency. A continuous theta-burst stimulation (cTBS) protocol adds two more parameters, the number of pulses in a burst and the burst frequency. Intermittent thetaburst stimulation (iTBS) protocols require a further two parameters. Here, the bursting protocol 
is applied for a given time (denoted ON time, often $2 \mathrm{~s}$ ), then removed for a given time (denoted OFF time, often $8 \mathrm{~s}$ ) before being active for another $2 \mathrm{~s}$ period, etc. Thus, the parameter space for describing intermittent bursting pulse sequences becomes vast. Experiments performed to date do not come close to spanning it.

\section{DESCRIPTIONS OF PREVIOUS RESULTS}

Recently, there has been some focus on developing good numerical models of the effects of rTMS (Robinson, 2011; Fung et al., 2013; Wilson et al., 2014). That has been our primary motivation for revisiting previous rTMS experiments (particularly cTBS and iTBS) with a view to identifying overall trends. Which parameters are the most important? What, on average, are their effects? Various forms of statistical regression, such as Principal Component Analysis (PCA) should potentially be of benefit here. However, it quickly became clear that for bursting sequences (e.g., cTBS and iTBS) there is insufficient variation in data for such an analysis to be meaningful. Few protocols have been evaluated in the large parameter space. Recently, for example, the cTBS and iTBS protocols of Huang et al. (2005) have come to the fore and are being used to the substantial exclusion of other possibilities.

In the case of repetitive, but not bursting protocols, there is more variation, particularly in earlier studies. This has allowed us to tackle an analysis with regression and PCA. We have identified 92 publications looking at rTMS protocols (from 1994 to 2006) containing results of 164 different experiments. However, in many of these cases data were not systematically reported and key information was missing. From these publications we produced a subset of 35 publications containing data from 79 different experiments in which we could unambiguously identify the frequency of the rTMS, the number of pulses applied in the protocol, the intensity of the applied pulse (in terms of \%RMT) and the overall effect on the MEP. These experiments covered 1064 different subjects (although some of these subjects are likely to be the same person). The number of subjects in these experiments ranged from four to 45 , with a median of ten. Nineteen publications reported on "one-off" experiments with a single protocol, as opposed to multiple protocols used on the same set of subjects. Various clinical populations were covered, including Parkinson's disease, epilepsy, major depression and focal hand dystonia, in addition to healthy populations. We have not looked at the effect of population on results.

A few publications recorded the effect on the MEP in a quantitative manner; most did not. Rather, it has been usually recorded qualitatively as "increase," "decrease," "no change," or, unhelpfully, "variable." Again, there is a problem of interpretation here. What is it about the MEP that "increases"? Is it the amplitude of the MEP, the time-scale over which it occurs, or the integrated area of the MEP? Many publications are vague on this point. We have taken a pragmatic approach and simply left the effect on the MEP, for the purposes of analysis, as being "increase," "decrease" or "no change." We assigned each of these results the numeric values of 1,0 , and -1 , respectively.
Those protocols which recorded "variable" results, were assigned 0 , although the results of analyses do not change much when these experiments are excluded.

We thus constructed a four-dimensional dataset recording frequency of rTMS $(f)$, number of pulses applied $(N)$, amplitude of each pulse $(A)$, and (tri-valued) effect on the MEP, $M$. We then carried out two analyses. First, we used linear regression to find the overall effect of $f, N$, and $A$ on $M$. Secondly, we used PCA to determine which variable combinations were most important for influencing $M$ and their overall effect. To eliminate some bias, we weighted each experiment by the number of participants.

The regression analysis showed that it was only the pulse frequency $f$ that had any overall effect on the size of the MEP. For frequency, the analysis is shown in Figure 1A. Here, the result is plotted against the applied frequency. The area of the circles is proportional to the number of subjects in each experiment. The solid line shows the results of linear regression (with each experiment weighted by the number of participants); the dashed line shows regression where the more questionable "one-off" experiments have been excluded. We acknowledge that linear regression is dubious since we have made the results tri-valued only and there is no a priori reason for believing a linear relationship should apply.

Results of PCA are summarized in Figure 1B. From a fourdimensional data-set we obtain four principal components. The components, in order, explain $42,31,16$, and $11 \%$ of the variation in the data. We plot the data points in terms of the first two principal components, and show the direction of the $f, N, A$ and $M$ axes on the figure.

It is clear that frequency is the major driver of the change in the MEP. Figure 1A shows the effect is for low frequencies to reduce the MEP, and high frequencies to increase it, in line with the much-assumed $f<5 \mathrm{~Hz}$, depression; $f>5 \mathrm{~Hz}$, potentiation. However, the relationship is weak, with results widespread. This is supported by Figure 1B, which indicates that the $M$ axis lies in a similar direction to the $f$ axis, meaning that an increase in $f$ leads in general to an increase in the end result $M$. Results show little link between $N$ and $A$ and the end result. On the PCA plot of Figure 1B, the $N$ and $A$ axes lie roughly perpendicular to the end result. Linear regression (not shown) gives no apparent trend.

\section{A REQUEST FOR MORE SYSTEMATIC DATA}

We do not wish to draw too much from the above results, other than to say that broadly speaking they support the established rTMS dogma around the effect of repetition rate on MEP. That in itself is unsurprising given that Fitzgerald et al. (2006) comprehensively tabulated a large number of experiments, and there is considerable overlap between our datasets. Too often, however, the mantra " $<5 \mathrm{~Hz}$, depression; $>5 \mathrm{~Hz}$, potentiation" is stated without acknowledging the extent of the variation in results. Although Héroux et al. (2015) have exposed some questionable research practices, 


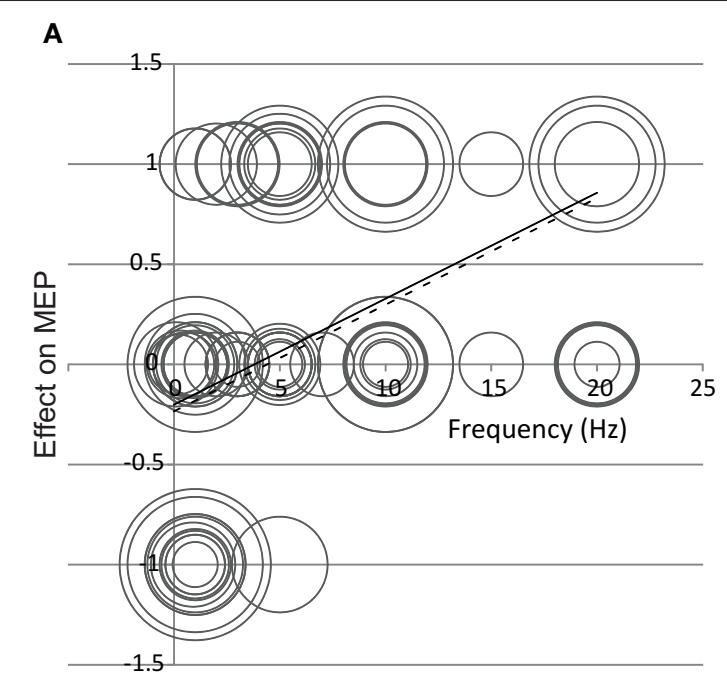

B

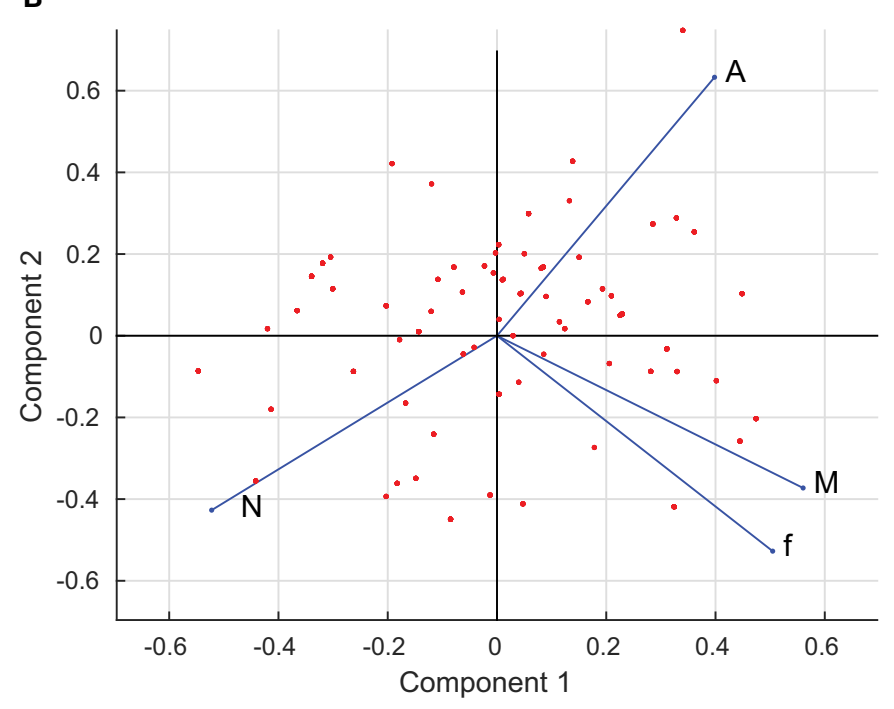

FIGURE 1 | (A) The effect of repetition frequency $f$ on the size of the MEP. The circles denote different experiments; the area of the circles is proportional to the number of participants in each experiment. The solid line is a result of linear regression; the dashed line is a result of linear regression where "one-off" experiments have been excluded. (B) A two-dimensional rendering of the rTMS data set using Principal Component Analysis. The first and second components form the $x$ - and $y$-axes respectively. The points mark the individual experiments. The $f, N, A$, and $M$ axes are shown in terms of the first two principal components. Data are taken from: Pascual-Leone et al., 1994; Jennum et al., 1995; Wassermann et al., 1996; Chen et al., 1997a,b; Berardelli et al., 1998, 1999; Siebner et al., 1999a,b; Maeda et al., 2000a,b; Muellbacher et al., 2000; Rollnik et al., 2000; Romeo et al., 2000; Siebner et al., 2000; Wu et al., 2000; Fierro et al., 2001; Lorenzano et al., 2002; Romero et al., 2002; Sommer et al., 2002; Cincotta et al., 2003; Gorsler et al., 2003; Grunhaus et al., 2003; Modugno et al., 2003; Schambra et al., 2003; Fitzgerald et al., 2004; Peinemann et al., 2004; Stinear and Byblow, 2004; Brighina et al., 2005; Fitzgerald et al., 2005; Murase et al., 2005; Quartarone et al., 2005a,b; Daskalakis et al., 2006; Inghilleri et al., 2006.

excluding the "one-off" data makes little difference to this conclusion.

The main focus of our comments is the difficulty we experienced in performing such analyses, notably our inability to analyze the cTBS and iTBS literature in a quantitative manner.

First, we note that the typical output measure of rTMS, the MEP, is poorly defined. It is clear that different authors mean different things by this. It is not just a case that electrical activity of different muscles are measured; rather that there is no quantitative or even consistent qualitative definition of the change in the MEP. We are left mostly with vague terminology "increase," "decrease," "no change," or even "fluctuates." If we are to start understanding the results, drivers and mechanisms of rTMS, we need to start by robustly defining outcome measures. A minority of publications have focused on the length of the cortical silent period (CSP, the quiet period of the electromyogram following a MEP) rather than the "size" of the MEP. This is more easily quantifiable and is a more direct measure of cortical effects (Ziemann et al., 2015), therefore is possibly a better place to start. Unlike the MEP, which depends on a network of cortical and non-cortical excitatory and inhibitory processes, the CSP originates in the cortex and is mediated by activation of $\mathrm{GABA}_{\mathrm{A}}$ and $\mathrm{GABA}_{\mathrm{B}}$ (Ziemann et al., 2015).

If one persists with measuring the MEP, one needs also to relate changes in the MEP to changes in the brain. The response to TMS depends not only on cortical excitability but also to excitability at a spinal level and the properties of corticospinalmotoneurone connections. Without suitable models of the process, it is difficult to relate changes in MEP to changes in the cortex, and start to untangle the effects of plasticity, neural excitability and gene expression that all could affect the results of a TMS (Pell et al., 2011). One is therefore left with costly and difficult animal experiments (Vahabzadeh-Hagh et al., 2012).

Next, we must become more rigorous in the planning, execution, recording and publication of our experiments. Too often, key details have been left out. This has particularly been evident in some of the earlier experiments. In compiling our data set, we had to make assumptions about particular experiments because data were not unambiguously given. Indeed, our data set for producing the Figure used only around half of the literature we looked at, because we could not reliably identify the data we required. Moreover, our analysis relies on published data; we cannot analyze unpublished results.

Finally, we note that in the last 10 years human experiments have focused on a very limited range of pulse sequences, such as the cTBS and iTBS of Huang et al. (2005). Most of the high dimensioned parameter space that bursting rTMS sequences provide has been largely ignored. A much larger range of protocols is needed in order to investigate effects systematically. It is true that the focus on these protocols is a result of their promise in clinical applications, but in terms of 
science, we are left little the wiser about what rTMS is actually doing.

\section{AUTHOR CONTRIBUTIONS}

MW conceived the research, processed some of the data and wrote the majority of the article. LS identified the data set, carried

\section{REFERENCES}

Berardelli, A., Inghilleri, M., Gilio, F., Romeo, S., Pedace, F., Curra, A., et al. (1999). Effects of repetitive cortical stimulation on the silent period evoked by magnetic stimulation. Exp. Brain Res. 125, 82-86. doi: 10.1007/s002210050661

Berardelli, A., Inghilleri, M., Rothwell, J., Romeo, S., Curra, A., Gilio, F., et al. (1998). Facilitation of muscle evoked responses after repetitive cortical stimulation in man. Exp. Brain Res. 122, 79-84. doi: 10.1007/s002210050493

Brighina, F., Giglia, G., Scalia, S., Francolini, M., Palermo, A., and Fierro, B. (2005). Facilitatory effects of $1 \mathrm{~Hz}$ rTMS in motor cortex of patients affected by migraine with aura. Exp. Brain Res. 161, 34-38. doi: 10.1007/s00221-004-2042-7

Chen, R., Classen, J., Gerloff, C., Celnik, P., Wassermann, E., Hallett, M., et al. (1997a). Depression of motor cortex excitability by low-frequency transcranial magnetic stimulation. Neurology 48, 1398-1403.

Chen, R., Samii, A., Canos, M., Wassermann, E., and Hallett, M. (1997b). Effects of phenytoin on cortical excitability in humans. Neurology 49, 881-883.

Cincotta, M., Borgheresi, A., Gambetti, C., Balestrieri, F., Rossi, L., Zaccara, G., et al. (2003). Suprathreshold $0.3 \mathrm{~Hz}$ repetitive TMS prolongs the cortical silent period: potential implications for therapeutic trials in epilepsy. Clin. Neurophysiol. 114, 1827-1833. doi: 10.1016/S1388-2457(03)00181-0

Daskalakis, Z. J., Möller, B., Christensen, B. K., Fitzgerald, P. B., Gunraj, C., and Chen, R. (2006). The effects of repetitive transcranial magnetic stimulation on cortical inhibition in healthy human subjects. Exp. Brain Res. 174, 403-412. doi: 10.1007/s00221-006-0472-0

Fierro, B., Piazza, A., Brighina, F., La Bua, V., Buffa, D., and Oliveri, M. (2001). Modulation of intracortical inhibition induced by low-and high-frequency repetitive transcranial magnetic stimulation. Exp. Brain Res. 138, 452-457. doi: $10.1007 / \mathrm{s} 002210100728$

Fitzgerald, P. B., Benitez, J., Oxley, T., Daskalakis, J. Z., de Castella, A. R., and Kulkarni, J. (2005). A study of the effects of lorazepam and dextromethorphan on the response to cortical $1 \mathrm{~Hz}$ repetitive transcranial magnetic stimulation. Neuroreport 16, 1525-1528. doi: 10.1097/01.wnr.0000177005.14108.f1

Fitzgerald, P. B., Brown, T. L., Marston, N. A., Oxley, T., de Castella, A., Daskalakis, Z. J., et al. (2004). Reduced plastic brain responses in schizophrenia: a transcranial magnetic stimulation study. Schizophr. Res. 71, 17-26. doi: 10.1016/j.schres.2004.01.018

Fitzgerald, P. B., Fountain, S., and Daskalakis, Z. J. (2006). A comprehensive review of the effects of rTMS on motor cortex excitability and inhibition. Clin. Neurophysiol. 117, 2584-2596. doi: 10.1016/j.clinph.2006.06.712

Fregni, F., Simon, D. K., Wu, A., and Pascual-Leone, A. (2005). Non-invasive brain stimulation for Parkinson's disease: a systematic review and metaanalysis of the literature. J. Neurol. Neurosurg. Psychiatry 76, 1614-1623. doi: 10.1136/jnnp.2005.069849

Fung, P. K., Haber, A. L., and Robinson, P. A. (2013). Neural field theory of plasticity in the cerebral cortex. J. Theor. Biol. 318, 44-57. doi: 10.1016/j.jtbi.2012.09.030

Gorsler, A., Bäumer, T., Weiller, C., Münchau, A., and Liepert, J. (2003). Interhemispheric effects of high and low frequency rTMS in healthy humans. Clin. Neurophysiol. 114, 1800-1807. doi: 10.1016/S1388-2457(03)00157-3

Grunhaus, L., Polak, D., Amiaz, R., and Dannon, P. N. (2003). Motorevoked potential amplitudes elicited by transcranial magnetic stimulation do not differentiate between patients and normal controls. Int. J. Neuropsychopharmacol. 6, 371-378. doi: 10.1017/S1461145703 003705

Hallet, M. (2007). Transcranial magnetic stimulation: a primer. Neuron 55, 187199. doi: 10.1016/j.neuron.2007.06.026 out most of the data processing, identified the major issues and wrote the minority of the article.

\section{ACKNOWLEDGMENTS}

LS was funded by the University of Waikato Summer Scholarship Programme 2015/2016.

Hamada, M., Murase, N., Hasan, A., Balaratnam, M., and Rothwell, J. C. (2013). The role of interneuron networks in driving human motor cortical plasticity. Cereb. Cortex 23, 1593-1605. doi: 10.1093/cercor/bhs147

Héroux, M. E., Taylor, J. L., and Gandevia, S. C. (2015). The use and abuse of transcranial magnetic stimulation to modulate corticospinal excitability in humans. PLoS ONE 10:e0144151. doi: 10.1371/journal.pone.0144151

Huang, Y.-Z., Edwards, M. J., Rounis, E., Bhatia, K. P., and Rothwell, J. (2005). Theta burst stimulation of the human motor cortex. Neuron 45, 201-206. doi: 10.1016/j.neuron.2004.12.033

Inghilleri, M., Conte, A., Frasca, V., Scaldaferri, N., Gilio, F., Santini, M., et al. (2006). Altered response to rTMS in patients with alzheimer's disease. Clin. Neurophysiol. 117, 103-109. doi: 10.1016/j.clinph.2005.09.016

Jennum, P., Winkel, H., and Fuglsang-Frederiksen, A. (1995). Repetitive magnetic stimulation and motor evoked potentials. Electroencephalogr. Clin. Neurophysiol. Electromyogr. Motor Control 97, 96-101. doi: 10.1016/0924980X(94)00293-G

Loo, C. K., and Mitchell, P. B. (2005). A review of the efficacy of transcranial magnetic stimulation (TMS) treatment for depression, and current and future strategies to optimize efficacy. J. Affect. Disord. 88, 255-267. doi: 10.1016/j.jad.2005.08.001

Lorenzano, C., Gilio, F., Inghilleri, M., Conte, A., Fofi, L., Manfredi, M., et al. (2002). Spread of electrical activity at cortical level after repetitive magnetic stimulation in normal subjects. Exp. Brain Res. 147, 186-192. doi: 10.1007/s00221-002-1237-z

Maeda, F., Keenan, J. P., Tormos, J. M., Topka, H., and Pascual-Leone, A. (2000a). Interindividual variability of the modulatory effects of repetitive transcranial magnetic stimulation on cortical excitability. Exp. Brain Res. 133, 425-430. doi: $10.1007 / \mathrm{s} 002210000432$

Maeda, F., Keenan, J. P., Tormos, J. M., Topka, H., and Pascual-Leone, A. (2000b). Modulation of corticospinal excitability by repetitive transcranial magnetic stimulation. Clin. Neurophysiol. 111, 800-805. doi: 10.1016/S13882457(99)00323-5

Modugno, N., Currà, A., Conte, A., Inghilleri, M., Fofi, L., Agostino, R., et al. (2003). Depressed intracortical inhibition after long trains of subthreshold repetitive magnetic stimuli at low frequency. Clin. Neurophysiol. 114, 24162422. doi: 10.1016/S1388-2457(03)00262-1

Muellbacher, W., Ziemann, U., Boroojerdi, B., and Hallett, M. (2000). Effects of low-frequency transcranial magnetic stimulation on motor excitability and basic motor behavior. Clin. Neurophysiol. 111, 1002-1007. doi: 10.1016/S13882457(00)00284-4

Murase, N., Rothwell, J. C., Kaji, R., Urushihara, R., Nakamura, K., Murayama, N., et al. (2005). Subthreshold low-frequency repetitive transcranial magnetic stimulation over the premotor cortex modulates writer's cramp. Brain 128, 104-115. doi: 10.1093/brain/awh315

Nettekoven, C., Vlotz, L. J., Leimback, M., Pool, E.-M., Rehme, A. K., Eickhoff, S. B., et al. (2015). Inter-individual variability in cortical excitability and motor network connectivity following multiple blocks of rTMS. Neuroimage 118, 209-218. doi: 10.1016/j.neuroimage.2015.06.004

Nicolo, P., Ptak, R., and Guggisberg, A. G. (2015). Variability of behavioural responses to transcranial magnetic stimulation: origins and predictors. Neurophsychlogia 74, 137-144. doi: 10.1016/j.neuropsychologia.2015. 01.033

O’Reardon, J. P., Solvason, H. B., Janicak, P. G., Sampson, S., Isenberg, K. E., Nahas, Z., et al. (2007). Efficacy and safety of transcranial magnetic stimulation in the acute treatment of major depression: a multisite randomized control trial. Biol. Psychiatry 62, 1208-1216. doi: 10.1016/j.biopsych.2007.01.018 
Pascual-Leone, A., Valls-Solé, J., Wassermann, E. M., and Hallett, M. (1994). Responses to rapid-rate transcranial magnetic stimulation of the human motor cortex. Brain 117, 847-858. doi: 10.1093/brain/117.4.847

Peinemann, A., Reimer, B., Löer, C., Quartarone, A., Münchau, A., Conrad, B., et al. (2004). Long-lasting increase in corticospinal excitability after 1800 pulses of subthreshold $5 \mathrm{~Hz}$ repetitive TMS to the primary motor cortex. Clin. Neurophysiol. 115, 1519-1526. doi: 10.1016/j.clinph.2004.02.005

Pell, G. S., Roth, Y., and Zangen, A. (2011). Modulation of cortical excitability induced by repetitive transcranial magnetic stimulation: influence of timing and geometrical parameters and underlying mechanisms. Prog. Neurobiol. 93, 59-98. doi: 10.1016/j.pneurobio.2010.10.003

Quartarone, A., Bagnato, S., Rizzo, V., Morgante, F., SantAngelo, A., Battaglia, F., et al. (2005a). Distinct changes in cortical and spinal excitability following high-frequency repetitive TMS to the human motor cortex. Exp. Brain Res. 161, 114-124. doi: 10.1007/s00221-004-2052-5

Quartarone, A., Rizzo, V., Bagnato, S., Morgante, F., Sant'Angelo, A., Romano, M., et al. (2005b). Homeostatic-like plasticity of the primary motor hand area is impaired in focal hand dystonia. Brain 128, 1943-1950. doi: 10.1093/brain/awh527

Ridding, M. C., and Rothwell, J. C. (2007). Is there a future for therapeutic use of transcranial magnetic stimulation. Nat. Rev. Neurosci. 8, 559-567. doi: $10.1038 / \mathrm{nrn} 2169$

Robinson, P. A. (2011). Neural field theory of synaptic plasticity. J. Theor. Biol. 285, 156-163. doi: 10.1016/j.jtbi.2011.06.023

Rollnik, J. D., Schubert, M., and Dengler, R. (2000). Subthreshold prefrontal repetitive transcranial magnetic stimulation reduces motor cortex excitability. Muscle Nerve 23, 112-114. doi: 10.1002/(SICI)10974598(200001)23:1<112::AID-MUS15>3.0.CO;2-B

Romeo, S., Gilio, F., Pedace, F., Ozkaynak, S., Inghilleri, M., Manfredi, M., et al. (2000). Changes in the cortical silent period after repetitive magnetic stimulation of cortical motor areas. Exp. Brain Res. 135, 504-510. doi: $10.1007 / \mathrm{s} 002210000541$

Romero, J. R., Anschel, D., Sparing, R., Gangitano, M., and PascualLeone, A. (2002). Subthreshold low frequency repetitive transcranial magnetic stimulation selectively decreases facilitation in the motor cortex. Clin. Neurophysiol. 113, 101-107. doi: 10.1016/S1388-2457(01) 00693-9

Schambra, H., Sawaki, L., and Cohen, L. (2003). Modulation of excitability of human motor cortex (M1) by $1 \mathrm{~Hz}$ transcranial magnetic stimulation of the contralateral M1. Clin. Neurophysiol. 114, 130-133. doi: 10.1016/S13882457(02)00342-5

Siebner, H., Tormos, J., Ceballos-Baumann, A., Auer, C., Catala, M., Conrad, B., et al. (1999a). Low-frequency repetitive transcranial magnetic stimulation of the motor cortex in writer's cramp. Neurology 52, 529-529.

Siebner, H. R., Auer, C., and Conrad, B. (1999b). Abnormal increase in the corticomotor output to the affected hand during repetitive transcranial magnetic stimulation of the primary motor cortex in patients with writer's cramp. Neurosci. Lett. 262, 133-136.
Siebner, H. R., Mentschel, C., Auer, C., Lehner, C., and Conrad, B. (2000) Repetitive transcranial magnetic stimulation causes a short-term increase in the duration of the cortical silent period in patients with Parkinson's disease. Neurosci. Lett. 284, 147-150. doi: 10.1016/S0304-3940(00)00990-3

Sommer, M., Lang, N., Tergau, F., and Paulus, W. (2002). Neuronal tissue polarization induced by repetitive transcranial magnetic stimulation? Neuroreport 13, 809-811. doi: 10.1097/00001756-200205070-00015

Stinear, C. M., and Byblow, W. D. (2004). Impaired modulation of corticospinal excitability following subthreshold rTMS in focal hand dystonia. Human Movem. Sci. 23, 527-538. doi: 10.1016/j.humov.2004.08.022

Thut, G., and Pascual-Leone, A. (2010). A review of combined TMS-EEG studies to characterize lasting effects of repetitive TMS and assess their usefulness in cognitive and clinical neuroscience. Brain Topogr. 22, 219-232. doi: 10.1007/s10548-009-0115-4

Tsutsumi, R., Hanajima, R., Terao, Y., Shirota, Y., Ohminami, S., Shimizu, T., et al. (2014). Effects of the motor cortical quadripulse transcranial magnetic stimulation (QPS) on the contralateral motor cortex and interhemispheric interactions. J. Neurophysiol. 111, 26-35. doi: 10.1152/jn.00515.2013

Vahabzadeh-Hagh, A. M., Muller, P. A., Gersner, R., Zangen, A., and Rotenberg, A. (2012). Translational neuromodulation: approximating human transcranial magnetic stimulation protocols in rats. Neuromodulation 15, 296-305. doi: 10.1111/j.1525-1403.2012.00482.x

Wassermann, E. M., Grafman, J., Berry, C., Hollnagel, C., Wild, K., Clark, K., et al. (1996). Use and safety of a new repetitive transcranial magnetic stimulator. Electroencephalogr. Clin. Neurophysiol. Electromyogr. Motor Control 101, 412417. doi: 10.1016/0924-980X(96)96004-X

Wilson, M. T., Goodwin, D. P., Brownjohn, P. W., Shemmell, J., and Reynolds, J. N. J. (2014). Numerical modelling of plasticity induced by transcranial magnetic stimulation. J. Comput. Neurosci. 36, 499-514. doi: 10.1007/s10827013-0485-1

Wu, T., Sommer, M., Tergau, F., and Paulus, W. (2000). Lasting influence of repetitive transcranial magnetic stimulation on intracortical excitability in human subjects. Neurosci. Lett. 287, 37-40. doi: 10.1016/S0304-3940(00) 01132-0

Ziemann, U., Reis, J., Schwenkreis, P., Rosanova, M., Strafella, A., Badawy, R., et al. (2015). TMS and drugs revisited 2014. Clin. Neurophysiol. 126, 1847-1868. doi: 10.1016/j.clinph.2014.08.028

Conflict of Interest Statement: The authors declare that the research was conducted in the absence of any commercial or financial relationships that could be construed as a potential conflict of interest.

Copyright (c) 2016 Wilson and St George. This is an open-access article distributed under the terms of the Creative Commons Attribution License (CC BY). The use, distribution or reproduction in other forums is permitted, provided the original author(s) or licensor are credited and that the original publication in this journal is cited, in accordance with accepted academic practice. No use, distribution or reproduction is permitted which does not comply with these terms. 